\title{
Centres de langues et politiques linguistiques européennes : une synergie?
}

\section{Nicole Poteaux}

\section{OpenEdition}

\section{Journals}

Édition électronique

URL : http://journals.openedition.org/asp/1212

DOI : 10.4000/asp. 1212

ISBN : 978-2-8218-0394-7

ISSN : 2108-6354

Éditeur

Groupe d'étude et de recherche en anglais de spécialité

Édition imprimée

Date de publication : 1 novembre 2003

Pagination : 115-123

ISSN : 1246-8185

Référence électronique

Nicole Poteaux, "Centres de langues et politiques linguistiques européennes : une synergie ? », ASp

[En ligne], 41-42 | 2003, mis en ligne le 28 avril 2010, consulté le 05 mai 2019. URL : http://

journals.openedition.org/asp/1212 ; DOI : 10.4000/asp.1212

Ce document a été généré automatiquement le 5 mai 2019

Tous droits réservés 


\title{
Centres de langues et politiques linguistiques européennes : une synergie?
}

\author{
Nicole Poteaux
}

1 Les centres de langues se sont développés de façon significative dans l'enseignement supérieur au cours des quinze dernières années. Les cours de langues aux étudiants spécialistes d'autres disciplines ont évolué vers d'autres formes d'organisation et de contenus. Nous ferons l'hypothèse que ces changements répondent à des évolutions sociales, politiques, économiques dont l'influence n'est pas toujours immédiatement perceptible. D'autre part, si les recherches en linguistique et en didactique des langues ont traditionnellement des effets sur l'enseignement des langues, d'autres domaines comme la sociologie, la psychologie du développement, la philosophie, les sciences de l'éducation, l'informatique ont aussi peu ou prou influencé les développements récents de l'apprentissage des langues. Étant donné la multiplicité des influences et des discours, il est difficile d'identifier les liens qui unissent la conception de centres de langues aux différents domaines de recherche concernés. En même temps, l'évolution des politiques linguistiques européennes produit un discours en pleine mutation sur l'enseignement des langues. Les mises en œuvre préconisées renvoient à une organisation de l'enseignement/ apprentissage de type «centre de langues ${ }^{1}$ ». Nous entendons par là que les étudiants sont évalués sur des compétences qu'ils auront travaillées à leur façon et non pas sur les contenus des cours qu'ils auront suivis. Cette vision de l'évaluation constitue peut-être actuellement un frein autant matériel que psychologique à l'avancement du projet "CLES ». De même, la conception de la maîtrise des langues et du plurilinguisme évolue au sein des institutions européennes. ${ }^{2}$ Des convergences apparaissent nettement, mais dans quelle mesure les enseignants qui s'impliquent dans la création de structures nouvelles dans leurs institutions prennent-ils consciemment ces enjeux en compte?

Dans ce texte, nous tenterons de repérer les articulations qui s'opèrent dans les secteurs politiques, sociaux et didactiques. Dans un premier temps nous considérerons les 
recherches dans le domaine de l'apprentissage intervenant dans la conception des centres de langues. Puis nous pointerons à travers les textes récents des institutions européennes les convergences avec les éléments développés précédemment pour mieux identifier la dynamique actuelle de l'enseignement des langues dans l'enseignement supérieur.

3 Il n'est pas question de définir ici la notion de « centre de langues ». Nous nous y sommes déjà essayé3. D'autres s'y sont attelés depuis ${ }^{4}$. Nous nous contenterons d'en rappeler les traits caractéristiques et fédérateurs. Qu'ils allient cours et travail en salles de ressources, qu'ils accordent une part plus ou moins importante au développement de l'autonomie de l'étudiant, que la technologie y occupe une plus ou moins grande place, les centres de langues sont conçus pour fournir à leurs étudiants des environnements riches en ressources pédagogiques variées afin d'offrir une efficacité maximale pour l'apprentissage et la pratique des langues étrangères. Ils ont pour objectif la recherche de moyens nouveaux adaptés aux publics actuels de l'enseignement supérieur et à la demande sociale de formation. L'intégration des technologies de l'information et de la communication caractérise également les initiatives prises dans les centres de langues; l'utilisation du multimédia et des réseaux informatiques pour l'apprentissage des langues en constitue un secteur de recherche important. Dans le même temps et dans une large mesure, ils intègrent la notion de développement de l'autonomie de l'étudiant en privilégiant les parcours d'apprentissage individuels et l'apprentissage auto-dirigé. Contrairement à certaines craintes des enseignants ou à certaines velléités institutionnelles, le rôle des enseignants reste primordial, même s'ils sont amenés à modifier leurs pratiques pédagogiques habituelles pour se consacrer davantage à des tâches de création de supports pédagogiques ainsi qu'à des activités de suivi des étudiants sous forme de conseil méthodologique et d'accompagnement. En conséquence, les recherches qui y sont menées prennent pour objet, d'une part, les conditions de création de dispositifs pédagogiques innovants et, d'autre part, leurs effets sur l'apprentissage.

4 Ayant développé à grande échelle à l'université de Strasbourg l'apprentissage en centres de ressources de langues ${ }^{5}$, nous appuierons plus particulièrement notre réflexion sur notre expérience (Bucher-Poteaux 1998, Poteaux 2000) et sur des résultats de recherche issus de notre terrain d'expérimentation. La spécificité du modèle strasbourgeois réside dans le fait que l'apprentissage et la pratique des langues sont fondés sur le développement de l'autonomie des étudiants dès la première année à l'université. Le travail des enseignants s'effectue principalement dans le suivi de l'étudiant ainsi que dans l'organisation des ressources pédagogiques. Le dispositif actuel est le résultat d'une série de choix méthodologiques, eux-mêmes issus de données de recherche dans différents domaines disciplinaires. Il est entendu que nous ne pouvons prétendre à une généralisation de nos hypothèses aux autres centres de l'enseignement supérieur, mais nous savons, grâce aux congrès réguliers de nos associations, que les centres de langues se rejoignent sur les principaux axes méthodologiques.

\section{Le modèle de l'activité humaine}

Nous prendrons comme principales références Trocmé-Fabre $(1987,1999)$ et Linard $(1989$, 1996) qui, toutes deux linguistes au départ, ont cherché dans les théories de la connaissance humaine des pistes pour mieux comprendre les mécanismes cognitifs à l'œuvre dans l'apprentissage des langues ainsi que leur enjeu social. C'est en allant 
chercher du côté de la psychologie, expérimentale, sociale, et clinique, que Linard a trouvé les éléments qui lui ont permis de déplacer la perspective de "l'objet langue » à l'activité du sujet qui la parle. Il s'agit moins de travailler directement sur les éléments linguistiques qu'indirectement sur leurs conditions pratiques d'usage par les locuteurs, que ce soit au plan de la mobilisation de l'intention et de l'attention, de la discrimination des éléments essentiels, de l'énonciation en situation de dialogue et d'écriture ou de l'autocorrection. C'est ainsi qu'une approche pragmatique du langage rencontre nécessairement les théories de l'activité humaine élaborées dans d'autres disciplines. Les dimensions sociales et affectives de l'apprentissage, laissées au second plan dans le modèle de Piaget, ont été réhabilitées par Bruner (1996) qui, en définissant l'éducation comme "entrée dans la culture ", propose des modèles de la médiation humaine dans lesquels l'interaction entre enseignants et pairs est déterminante. Bruner insiste sur la nécessité de l'échange verbal afin d'externaliser le travail mental et de construire une communauté d'apprentissage ${ }^{6}$. Cependant la médiation par les objets matériels sera remise en avant par les théories de l'action de l'école russe de psychologie avec Vygotsky (1978) et Leontiev (1972) ${ }^{7}$. En faisant la synthèse de ces différents courants, M. Linard replace l'être humain dans ses dimensions corporelles, psycho-affectives, relationnelles :

En tant que phénomène du vivant, l'intelligence humaine est tributaire de l'espacetemps du corps et des relations psycho-affectives dans lesquelles elle prend naissance et se développe. En tant que système psycho-affectif interactif, elle se construit dans le jeu réciproque entre action effective sur le réel, seul moyen de validation, et abstraction réflexive, seul moyen de distanciation. Privilégier l'abstraction à l'excès, c'est développer le seul souci de la cohérence aux dépens de celui de la validation externe par l'épreuve du réel ; c'est faire de l'intelligence un estropié mégalomane, potentiellement dangereux. (1996:188)

6 Outre la réintroduction de la dimension affective dans l'apprentissage, dont le caractère crucial a été largement confirmé par les travaux de Damasio (1994), l'auteur redonne ainsi toute son importance à l'interaction sociale dans le développement de l'intelligence. La pluridimensionnalité de cette dernière demande de multiplier les modèles de référence comme les sources d'information et d'expériences, ces facteurs de développement d'aspect chaotique de la construction des connaissances. Enfin, une place doit être laissée à l'autonomie de chacun, tant sont importantes et imprévisibles les différences interindividuelles. Dans une perspective proche, Trocmé-Fabre propose que la pédagogie se remette à l'écoute des sciences du vivant et en tire les leçons pour l'apprentissage en considérant les stratégies avec lesquelles notre organisme s'est construit: la diversification, la complémentarité, l'échange et la coopération, la connexion, l'auto organisation, la sélectivité. Le couple percevoir-agir est fondateur du vivant : c'est dans l'interdépendance de ces deux processus cognitifs (perception, action) que la connaissance s'enracine.

Ce que je propose c'est que la pédagogie se mette à l'écoute des sciences du vivant. Ceci signifie qu'il est urgent que la pédagogie tienne compte de la réalité cognitive, des différents temps d'apprendre, des exigences de notre cerveau en matière d'équilibre, de recherche de sens, de connectivité, de flexibilité, d'auto organisation. (Trocmé-Fabre 1996 : 4)

7 En linguistique, nous trouverons chez Austin un écho à ces pensées : le faire et le dire sont imbriqués dans nos actions, nous faisons en disant, nous disons en faisant, en engageant notre expressivité de manière globale pour influer sur le cours des choses. Sur le plan pédagogique les conséquences sont limpides : si la situation dans laquelle se trouve une personne pour apprendre une langue ne lui permet pas de tisser le lien entre le faire et le 
dire, la langue restera une étrangeté virtuelle et ne prendra pas corps dans l'expérience de l'individu. D'où, dans cette perspective, l'importance du scénario comme décor de l'acte d'apprentissage, ou de la réalisation de projets, ou encore du jeu, comme le souligne Gaonac'h :

L'acquisition d'une langue seconde dépend de facteurs liés aux aspects communicationnels de la langue. [...] L'implication dans certaines activités à haut degré d'interaction sociale (le jeu par exemple), constitue un facteur éminemment positif de l'acquisition. L'existence d'une interaction effective nécessite une négociation des rôles, l'annonce d'intentions, l'expression d'avertissements, la planification d'actions, la sollicitation d'informations, la persuasion ou la contradiction, l'expression de demandes indirectes. (1991: 194-195)

Dans une réflexion menée sur le travail métacognitif, Narcy ${ }^{8}$ souligne également qu'une situation sociale donnée va conduire l'individu à réagir en fonction d'une émotion générée par cette situation; il se réfère également aux travaux de Damasio (1999) pour établir l'importance de l'intentionnalité de l'action.

En référence à ces travaux, l'apprentissage des langues, comme les autres apprentissages, est le résultat évolutif d'une auto construction par réorganisation des structures et des processus d'adaptation à l'environnement que l'individu possède dès avant sa naissance. Apprendre c'est d'abord agir et interagir avec son environnement et pour les humains, dans un milieu social et culturel qui en donne les motifs, les raisons et les moyens. Pour Vygotsky, l'action est d'abord un médiateur social, « une grammaire partagée de l'expérience ».

10 En respectant les caractéristiques individuelles des personnes telles que le rythme, les intérêts, la motivation, en donnant le choix des tâches et de leur planification, en organisant l'interaction avec les pairs et le dialogue pédagogique avec les enseignants, en instituant la réflexion sur l'apprentissage comme aide à l'apprentissage, en situant l'ordinateur comme processus de régulation qui permet à l'étudiant d'agir rapidement et d'avoir une information en retour sur son action, les centres de langues s'inscrivent totalement dans le modèle des théories de l'action. L'étudiant devient agent-acteur de ses propres intentions. L'apprentissage est considéré comme un processus complexe et progressif, autant individuel que social, rationnel qu'affectif, pratique que conceptuel.

11 Si la conception des centres de langues se fonde sur ces données théoriques, elle se différencie fondamentalement du modèle transmissif qui préside habituellement à l'organisation du système éducatif. Or, c'est dans l'enseignement supérieur, étape ultime du cursus scolaire que ce type de dispositif s'est le plus développé. On peut y voir le souci de se démarquer des longues années d'études secondaires qui n'ont pas toujours fait leurs preuves, ou la possibilité de profiter d'une plus grande souplesse dans l'organisation des études, ou la marque de la présence d'enseignants-chercheurs, ou encore la préoccupation de répondre au mieux à la professionnalisation proche des étudiants. En outre, au jour d'aujourd'hui, l'enjeu de la mobilité des étudiants est fortement lié à la construction d'un espace universitaire européen décidé par la déclaration de Bologne en 1999. Même si des expériences similaires voient le jour dans l'enseignement secondaire, elles restent cependant marginales alors qu'elles y auraient toute leur place. En revanche, l'avènement des Travaux Personnels Encadrés en lycée ou des Itinéraires de Découverte en collège se rapprochent d'une conception de l'apprentissage fondée sur le projet de l'élève et l'interdisciplinarité. Les langues étrangères y sont utilisées dans une perspective d'acquisition de connaissances dans d'autres disciplines ainsi que de communication. Si 
ces pratiques nouvelles se généralisent, elles influenceront peut-être à terme les futures générations de lycéens en modifiant leurs types d'acquisitions en langues étrangères.

\section{Les politiques européennes}

\subsection{La perspective actionnelle en didactique}

12 L'étude du Cadre Européen Commun de Référence (2001) faite par Puren (2002 : 55-71) montre l'évolution actuelle en matière de didactique des langues : elle s'accomplit dans une prise de distance par rapport à l'approche communicative pour former non plus seulement des interlocuteurs mais des acteurs sociaux :

La perspective privilégiée est de type actionnelle, en ce qu'elle considère avant tout l'usager et l'apprenant d'une langue comme des acteurs sociaux ayant à accomplir des tâches (qui ne sont pas seulement langagières) dans des circonstances et un environnement donné, à l'intérieur d'un domaine d'action particulier. Si les actes de parole se réalisent dans les activités langagières, celles-ci s'inscrivent elles-mêmes à l'intérieur d'actions en contexte social qui seules leur donnent leur pleine signification. [...] La perspective actionnelle prend donc aussi en compte les ressources cognitives, affectives, volitives et l'ensemble des capacités que possède et met en œuvre l'acteur social. (Cadre Européen Commun de Référence $2001: 15$ )

Il s'agit non plus de vivre ensemble avec nos différences mais de « faire ensemble », de coagir, notion qui se réfère là encore au constructivisme piagétien, à la primauté de l'interaction sociale de Bruner et tout simplement à la pédagogie de projet de Freinet.

On ne peut qu'être frappé par la convergence de ces déclarations d'ordre didactique avec les théories de l'activité humaine évoquées précédemment. Mais on peut aussi y voir la nécessité économique de former des individus amenés à travailler ensemble, et donc à agir ensemble, dans le cadre d'une mondialisation des échanges et des relations de travail. La didactique serait-elle sous influence?

Actuellement la commission Européenne intensifie la mobilisation pour l'apprentissage des langues et promeut la diversité linguistique en adoptant un plan d'action pour la période 2004-2006.

Pour pouvoir mieux profiter de la liberté de circulation dans le marché unique, pour être plus compétitifs dans l'économie de la connaissance, pour mieux connaître et comprendre les autres Européens dans une union élargie, les Européens doivent faire des efforts pour apprendre les langues. L'Année Européenne des langues 2001 a montré que nos concitoyens le souhaitent et le conseil européen de Barcelone en mars 2002, comme auparavant le Parlement européen, ont reconnu la nécessité d'agir. (Communiqué de presse de la Commission Européenne. Newspress 2002. <newspresspro.com>)

Cette déclaration est assortie d'une série de mesures qui vont de l'enseignement précoce à l'enseignement intégré en passant par l'organisation en 2006 d'une conférence européenne sur le recrutement des enseignants de langues. Nous retiendrons plus particulièrement la volonté d'élargir l'apprentissage des langues en le faisant sortir de la classe de langue par divers moyens : enseignement des disciplines en langues étrangères, encouragement au plurilinguisme, y compris les langues régionales et minoritaires, mise au point d'un indicateur européen de compétence linguistique en 2005 et 2006, projet de portail Internet d'information pour les professionnels et le grand public sur les formations disponibles, les avantages de parler plusieurs langues, etc. Par ces mesures 
qu'il déclare et soutient financièrement, le politique prend position : ce ne sont pas tant les langues comme objets d'étude qui l'intéressent, mais plutôt les gens qui parlent ces langues et qui doivent cohabiter en harmonie, travailler ensemble, échanger, faire preuve de mobilité, s'intéresser les uns aux autres. Cette force de décision dépasse celle des enseignants et des didacticiens qui, bon gré mal gré, exécuteront les décisions des instances politiques. L'histoire des méthodologies de l'enseignement des langues montre bien à travers les temps l'influence du politique sur l'enseignement: nous rappellerons pour mémoire et à titre d'exemple parmi de nombreux autres, qu'aux États-Unis, les laboratoires de langue ont émané directement de la nécessité de former la nation aux langues étrangères après le lancement du premier Spoutnik soviétique en 1957. Le National Defence Education Act de 1959 a promu les langues avec les sciences comme discipline d'intérêt national.

Actuellement, le Conseil de l'Europe prône une citoyenneté démocratique européenne qui se réaliserait, entre autres, dans une diversité linguistique et culturelle. La défense des langues et des cultures, toutes égales par ailleurs, vise aussi à combattre l'usage de l'anglais comme lingua franca et à instituer une Europe plurilingue. Contrairement à une idée longtemps répandue, apprendre une langue n'aurait plus comme suprême objectif la maitrise quasiment parfaite de l'idiome (Imagine you're English!) mais pourrait être remplacé par la maîtrise de compétences partielles dans plusieurs langues. L'avantage serait d'offrir à chacun la possibilité de parler sa langue en étant compris par bon nombre de ses concitoyens, étant soi-même capable de comprendre trois ou quatre autres langues. C'est dans cette perspective que des linguistes comme Blanche-Benveniste (1997) ont travaillé sur l'intercompréhension de langues de même origine. Dans la même optique, le potentiel linguistique d'un individu varie au cours de l'existence : les langues peuvent être en sommeil à certaines périodes, puis réactivées à certains moments; des compétences partielles peuvent s'acquérir rapidement et ponctuellement à des fins bien précises. Le citoyen européen se constituerait au cours de son existence un portefeuille de compétences qu'il gérerait au fil de ses intentions ou des nécessités de la vie.

Le couple traduction/valeurs de la méthodologie traditionnelle qui permettait de découvrir l'universalité des valeurs humaines à partir de textes choisis fait place à la découverte de valeurs communes par contact et confrontation à partir de l'interaction entre les individus. À titre d'exemple, nous avons constaté que ceux de nos étudiants qui travaillent en tandem ${ }^{9}$ et à qui nous demandons un compte-rendu commenté de leurs échanges, affirment acquérir quantité d'éléments culturels. Ils ne mentionnent pratiquement pas d'apprentissages linguistiques; ceux-ci semblent pourtant s'opérer à leur insu, toute leur attention et leur intention étant centrées sur la connaissance de l'autre et la recherche de valeurs partagées.

\subsection{Plurilinguisme et formation citoyenne}

La citoyenneté démocratique européenne ne se réduit pas à une forme de communication entre Européens. Le concept de citoyenneté est aussi de nature juridique, il appréhende les différents groupes ethniques sous les aspects des droits et des libertés. Il est clair que les langues sont concernées par cet enjeu. Seule une perspective plurilingue peut reconnaître et accepter la diversité linguistique. Si l'école, au sens large, relève le défi de participer activement à la constitution d'une identité européenne, constituée non par le partage de compétences linguistiques communes mais par l'adhésion à une valeur 
commune, soit une Europe plurilingue et pluriculturelle, elle devra revoir son offre de formation en langues. Il ne s'agira plus de considérer les langues comme une discipline parmi d'autres, organisées en langues maternelles, langues nationales, étrangères, secondes, régionales, mais comme une formation aux langues, voire à tous les langages, verbaux et non verbaux. L'éducation à la différence, l'éveil aux langues (Candelier 1998) et aux cultures étrangères dès le plus jeune âge seraient alors intégrés à la formation de base de tous les jeunes Européens. Lorsque les centres de langues rassemblent en un même lieu des langues diverses, des ressources variées et des publics différents, ils offrent, à petite échelle certes, la possibilité d'une pluralité de contacts linguistiques et culturels, de confrontation à la différence, de rencontres susceptibles de développer la curiosité et le goût pour des formes de plurilinguisme.

Des outils comme le Portfolio Européen des Langues (PEL) ont été conçus pour aider les individus à identifier leur bagage linguistique en termes de compétences et à l'accroître. Il leur donne la possibilité de prouver leurs progrès vers une compétence plurilingue enregistrant leurs expériences d'apprentissage de toute nature dans des langues variées, apprentissages dont la plupart ne pourraient faire l'objet de reconnaissance officielle. Le possesseur du PEL est amené à s'autoévaluer et à réfléchir à ses processus d'apprentissage, à se fixer des objectifs selon ses priorités. Le travail en centres de langues intègre facilement cet outil puisqu'il est conçu pour accompagner l'apprenant dans sa démarche personnelle. Les pratiques habituelles d'évaluation sont questionnées par cette stratégie innovante et suscitent des controverses. Pourtant le rapport final du projet pilote indique que $70 \%$ des apprenants estiment que le PEL les aide à évaluer leurs compétences. Notre expérience d'auto-évaluation (Poteaux 2003 : 134-135) montre qu'elle est un moyen de développement de l'autonomie des étudiants grâce à une pratique régulière. L'ensemble de cette démarche s'inscrit bien dans la prise de responsabilité de l'étudiant dans son apprentissage et son évaluation.

21 Les centres de langues se situent à un carrefour d'influences. Ils se démarquent de la pédagogie traditionnelle en s'inspirant de modèles d'apprentissage fondés sur les théories de l'action. Ils font usage des technologies de l'information et de la communication dans la mesure où elles s'inscrivent parfaitement dans cette perspective. Ils répondent à des demandes sociales actuelles de communication linguistique et culturelle à des fins professionnelles ou personnelles. Enfin, ils servent les projets de développement du plurilinguisme pour favoriser la mobilité dans une Europe démocratique et citoyenne. Autant les recherches sur la cognition sont relativement anciennes, autant les politiques linguistiques ont évolué récemment. On peut faire l'hypothèse que les secondes se sont inspirées, du moins en partie, des premières. Pourtant les références bibliographiques du Cadre Commun ou d'autres publications du Conseil de l'Europe font majoritairement référence à des linguistes anglo-saxons et à des didacticiens des langues. Rares sont les mentions de travaux en sociolinguistique ou en psychologie de l'apprentissage. Très peu de références sont faites à la question de l'apprentissage auto-dirigé. Pourtant les convergences existent bel et bien entre les préconisations politiques actuelles et une conception-organisation de l'apprentissage telle qu'elle se pratique dans les centres de langues. Il faut imaginer pour conclure que les enseignants de langue qui sont à l'initiative de dispositifs innovants ont eux-mêmes effectué la démarche de réflexion et d'appropriation de différents domaines de recherche pour les traduire en structures qui répondent à des impératifs sociaux-économiques mais qui restent puissamment fondées sur des principes de développement de l'individu à travers la pratique des langues 
étrangères. Ils sont les moteurs d'une dynamique qu'ils créent au moment où le contexte s'y prête, ce qui leur permet de rester garants des valeurs éducatives qu'ils défendent. Du moins, espérons le !

\section{BIBLIOGRAPHIE}

Austin, John. 1962. How to do things with words. Oxford : Oxford University Press.

Beacco, Jean-Claude et Michael Byram. 2003. Guide pour l'élaboration des politiques linguistiques en Europe. De la diversité linguistique à l'éducation plurilingue. Strasbourg : Conseil de l'Europe.

Blanche-Benveniste, C., A. Valli. 1997. «L'intercompréhension : le cas des langues romanes ». Le Français dans le monde. Numéro spécial.

Bruner, Jérôme. 1996. L'éducation, entrée dans la culture. Paris : Retz

Ucher-Poteaux, Nicole. 1998. « Des ressources... oui mais... pourquoi ? ». Études de Linguistique Appliquée 112, 483-494.

Candelier, Michel. 1998. «L'éveil aux langues à l'école primaire : le programme européen Evlang ». De la didactique des langues à la didactique du plurilinguisme, hommage à Louise Dabène. Grenoble : CD-LIDILEM, 299-308.

Conseil de L'Europe. 2001. Cadre Européen Commun de Référence pour les langues: Apprendre, Enseigner, Evaluer. Strasbourg : Conseil de l'Europe/Les éditions Didier.

Damasio, Antonio.1994, L'erreur de Descartes. La raison des émotions. Paris : Odile Jacob.

Damasio, Antonio. 1999. The feeling of what happens. Body and emotion in the making of consciousness .An Diego, CA : Harcourt Brace \& Co.

Gaonac'h, Daniel. 1991. Théories d'apprentissage et acquisition d'une langue étrangère. LAL

CREDIF. Paris : Hatier/Didier.

Leontiev, A. N. 1972. Le développement du psychisme, Traduction française ( $3^{\mathrm{e}}$ édition). Paris :

Éditions sociales.

Linard, Monique. 1996 [1989]. Des machines et des hommes. Apprendre avec les nouvelles technologies. 2

e édition. Paris : L'Harmattan.

Mathiot, Pascal. 2001. Singuliers passages. Essai sur la transmission des savoirs, Paris : Seuil.

Poteaux, Nicole. 2003. «L'autoformation à l'université : de quelques éléments dialectiques ». In Albero, B., Autoformation et enseignement supérieur. Paris : Hermès, 131-140.

Poteaux, Nicole. 2000. « Nouveaux dispositifs, nouvelles dispositions ». Les Langues Modernes 3, 8 12.

Puren, Christian. 2002. «Perspectives actionnelles et perspectives culturelles en didactique des langues-cultures : vers une perspective co-actionnelle et co-culturelle ». Les Langues Modernes 3, 55-71.

Trocmé-Fabre, Hélène, 1987. J'apprends, donc je suis. Paris : Éditions d'Organisation. 
Trocmé-Fabre, Hélène, 1996. « Apprendre aujourd'hui dans une université apprenante ». Centre international de recherches et études trans-disciplinaires. Bulletin 9.

Trocmé-Fabre, Hélène, 1999. Réinventer le métier d'apprendre. Paris : Éditions d'Organisation.

Vygotsky, Lev Semenovitch. 1978 [1934: $1^{\mathrm{e}}$ éd. Russe]. Mind in Society: the Development of Higher Psychological Functions. Cambridge, MA : Harvard University Press.

\section{NOTES}

1. Exemple : le décret du 22 mai 2000 instituant la Certification de Compétences en Langues de l'Enseignement Supérieur (CLES), fondé sur une évaluation des compétences décrites dans le Cadre Européen Commun de Référence, suppose un enseignement organisé en centre de langues.

2. Rappelons que les institutions européennes définissent des lignes directrices et élaborent des préconisations à destination des gouvernements des pays membres. Il est du ressort de chacun de les reprendre et de les diffuser selon sa propre politique.

3. Au sein de l'association RANACLES, la tentative de production d'un «Livre Blanc » des centres de langues s'est heurtée à la difficulté de rassembler l'énorme quantité d'informations nécessaires à une typologie ainsi qu'à la grande diversité des réalisations sur les différents terrains de l'enseignement supérieur.

4. Signalons un travail récent produit dans le cadre d'un projet SOCRATES (Lingua 1) en collaboration avec le Centre Européen des Langues Vivantes (CELV) de Graz qui répertorie les différents aspects en jeu dans les centres de ressources de langues, illustrés par les expériences des seize partenaires européens. <http://www.lrcnet.org/>

5. Actuellement six centres de ressources de langues fédérés en un Centre de langues et coordonnés pédagogiquement accueillent environ 12000 étudiants.

6. Cf. P. Mathiot : «La parole renvoie à la fois au langage comme passé, en s'appuyant sur des unités de son et de sens déjà constituées (phonèmes, morphèmes, sémantèmes) et renvoie au langage comme à quelque chose qui est encore à résulter, qui est toujours à venir, puisque le sens attend l'événement et la circonstance qui le rendront effectif, qui lui permettront de se constituer, de "prendre". [...] Inséparable d'un savoir acquis, elle est pourtant aussi une visée, une recherche en mouvement. Inséparable d'une expression et d'une reprise individuelle par un sujet qui se retrouve en elle, elle est aussi interpellation et discours d'adresse à l'autre et elle ne peut être construite qu'en faisant référence à une situation vécue et partagée d'interlocution qui, seule, donne sens à ce qu'elle désigne » (2001-61-62).

7. La théorie de l'activité soutient l'idée que le comportement d'un individu est gouverné, non seulement de l'intérieur, sur la base de ses fonctions biologiques et de ses fonctions psychologiques, mais aussi de l'extérieur, par la création et l'utilisation d'objets culturels appelés artefacts, instruments, machines, méthodes, qui jouent un rôle de médiateur entre le sujet et l'objet de son activité.

8. Conférence donnée au $10^{\mathrm{e}}$ congrès RANACLES « Pratiques et /ou Stratégies ? "Strasbourg, 28 novembre 2002.

9. Tandem Network met en relation à travers le monde des étudiants qui apprennent la langue l'un de l'autre. < http://www.cisi.unito.it/tandem/email/idxeng00.html> 


\section{RÉSUMÉS}

Apprendre et pratiquer les langues étrangères dans l'enseignement supérieur aujourd'hui s'inscrit dans un contexte politique, économique et social qui influence les pratiques pédagogiques. Les politiques linguistiques européennes affichent une volonté de développement qui questionne les pratiques en vigueur. D'autre part, les recherches sur l'apprentissage en font évoluer la conception. La question est de savoir comment se situent les centres de langues dans ce réseau d'influences et sur quelles bases ils se fondent pour se développer.

Political, social and economic context influence the way foreign languages are learnt and used in higher education. The European policy of promoting languages has led us to question traditional teaching methods. In addition, research has changed our perception of the learning process. How do these factors influence the development of language centres?

\section{INDEX}

Keywords : autonomy, Europe, language centre, learning, linguistic policy, plurilinguism Mots-clés : apprentissage, autonomie, centre de langues, plurilinguisme, politique linguistique

\section{AUTEUR}

\section{NICOLE POTEAUX}

Nicole Poteaux est maître de conférences en Sciences de l'éducation à l'Université Louis Pasteur de Strasbourg. Angliciste de première formation, elle s'intéresse aux questions d'apprentissage des langues en général et à l'apprentissage auto-dirigé en particulier. Responsable de l'enseignement des langues, elle coordonne sept centres de ressources de langues et continue ses recherches sur l'évaluation des dispositifs ainsi que sur la construction des parcours d'apprentissage par les étudiants. nicole.poteaux@lse-ulp.u-strasbg.fr 